\title{
DESENVOLVIMENTO DE COMPETÊNCIAS DE PROMOÇÃO DA SAÚDE NO PROJETO PRAIA SEM BARREIRAS
}

\author{
Fabiana Gonçalves de Mello Cahú ${ }^{1}$, Fellipe Eugênio Soares Miranda ${ }^{1}$, Ana Claudia \\ Camargo Gonçalves Germani ${ }^{2}$, Maíra Rosa Apostólico ${ }^{3}$ e Alfredo Almeida Pina-Oliveira ${ }^{3}$ \\ ${ }^{1}$ Centro Universitário Maurício de Nassau (UNINASSAU), Brasil. fabianacahu@hotmail.com; \\ fellipesoaresmiranda@gmail.com \\ 2Universidade de São Paulo (FMUSP-SP), Brasil. ana.germani@fm.usp.br \\ ${ }^{3}$ Universidade Guarulhos (UNIVERITAS-UNG), Brasil. maira.apostolico@prof.ung.br; aapo_enf@yahoo.com.br
}

\begin{abstract}
Resumo. Introdução: Promover a saúde das pessoas com diferentes tipos de deficiência torna-se relevante e desafiador para a graduação em saúde. Nesse sentido, o Projeto Praia sem Barreiras representa uma ação de extensão universitária interprofissional e intersetorial com potencial para o desenvolvimento de competências de promoção da saúde dos profissionais que nele atuam. Objetivo: Analisar a compreensão dos usuários do projeto sobre as práticas de Promoção da Saúde desenvolvidas pelos estudantes de graduação em saúde. Método: Estudo exploratório com abordagem qualitativa, baseado em entrevistas semiestruturadas com dez usuários do PSB da praia de Boa Viagem, Recife, Pernambuco, Brasil. Adotou-se a modalidade temática da análise de conteúdo e o referencial teórico-operacional do Developing Competencies and Profissional Standards for Health Promotion Capacity Building in Europe. Resultados: Os usuários do projeto indicaram a necessidade de qualificação dos acadêmicos para promover a saúde de pessoas com deficiência ou mobilidade reduzida com mais segurança. Evidenciaram-se os domínios possibilidade de mudanças, comunicação e parceria na atuação discente no projeto. Considerações finais: Outros domínios do referencial teórico devem ser aprofundados na graduação, para desenvolver conhecimentos, habilidades e valores essenciais para promover a saúde das pessoas com deficiência ou mobilidade reduzida.
\end{abstract}

Palavras-chave: Promoção da Saúde; Pessoas com Deficiência; Educação Baseada em Competências; Estudantes de Ciências da Saúde; Relações Comunidade-Instituição.

\section{DEVELOPING HEALTH PROMOTION COMPETENCIES IN THE PRAIA SEM BARREIRAS PROJECT}

\begin{abstract}
Introduction: Promoting the health of people with different types of disabilities becomes relevant and challenging for health graduates. In this sense, the "Praia sem Barreiras" Project represents an action for developing health promotion competencies. Objective: To analyse the project users' understanding of Health Promotion practices developed by undergraduate health students. Method: Exploratory study with a qualitative approach, based on semi-structured interviews with ten PSB users from Boa Viagem beach, Recife, Pernambuco, Brazil. The thematic modality of content analysis and the theoretical-operational framework of the Developing Competencies and Professional Standards for Health Promotion Capacity Building in Europe were adopted. Results: The users of the project indicated the need for qualification of academics to promote the health of people with disabilities or reduced mobility more safely. We evinced the domains of enabling change, communication and partnership related to student's performance in the project. Final considerations: Other domains of the theoretical framework must be deepened in graduation, to develop knowledge, skills and essential values to promote the health of people with disabilities or reduced mobility.
\end{abstract}

Keywords: Health Promotion; Disabled Persons; Competency-Based Education; Health Sciences Students; Community-Institutional Relations. 


\section{INTRODUÇÃO}

A inclusão social é um valor fundante da atual Política Nacional de Promoção da Saúde no contexto brasileiro e que deve ser considerado em ações fundamentadas no conceito positivo e ampliado de saúde. Nesse sentido, o desenvolvimento de iniciativas de modificação arquitetônicas e de mobilidade urbana objetivem a garantia de acesso às pessoas com deficiência (PCD), com mobilidade reduzida e ou idosas, buscando garantir os direitos humanos e as liberdades fundamentais (Brasil, 2014, Baptista \& Silva, 2015). Muitas conquistas internacionais e nacionais fortaleceram o avanço dos direitos humanos e, em particular, das PCD (Brasil, 1988; ONU, 2006; Garcia, 2011; Lanna \& Martins, 2010, Brasil, 2015) e a legislação brasileira integrou leis mais específicas (Lanna \& Martins, 2010; Brasil, 2015). Em âmbito internacional, a expressão PCD foi usada e consagrada pela Convenção sobre os Direitos das Pessoas com Deficiência e homologada em 2006 pela Organização das Nações Unidas, ONU (ONU, 2006).

Essa Convenção preconiza a acessibilidade como princípio, sendo condição para a garantia de todo e qualquer direito humano (Bezerra, 2014). As pessoas com deficiência apresentam impedimentos de longo prazo de natureza física, mental, intelectual ou sensorial, sendo que tais barreiras comprometem sua participação plena e efetiva na sociedade em igualdades de condições com as demais pessoas (ONU, 2006).

Por outro lado, considera-se pessoa com mobilidade reduzida: "aquela que, não se enquadrando no conceito de pessoa portadora de deficiência, tenha, por qualquer motivo, dificuldade de movimentar-se, permanente ou temporariamente, gerando redução efetiva da mobilidade, flexibilidade, coordenação motora e percepção" (Brasil, 2004, p. 2).

No Brasil, o planejamento e a urbanização das praias devem ser concebidos e executados de forma a torná-las acessíveis para as PCD ou com mobilidade reduzida (Brasil, 2000; Ruschmann, 2006), permitindo uma ampla acessibilidade a estes cidadãos, diminuindo os obstáculos à sua participação e à melhor utilização em situação de inclusão social (Mazzota \& Dantino, 2011).

Estima-se que 15,6\% da população mundial possuam algum tipo de deficiência (ONU, 2011). No último censo realizado em 2010, o Instituto Brasileiro de Geografia e Estatística (IBGE) indicou que 45,6 milhões de pessoas, representadas por $23,9 \%$ da população brasileira, apresentava pelo menos um tipo de deficiência, seja do tipo visual, auditiva, motora ou mental 
ou intelectual. A região Nordeste do Brasil concentrava $26,3 \%$ desta população, sendo que os estados da Paraíba e do Rio Grande do Norte apresentavam a maior prevalência de PCD. O estado de Pernambuco ocupava o terceiro lugar e apresentava $27,5 \%$ de PCD na sua população à época (IBGE, 2010).

Iniciativas para as PCD despontam em diferentes cenários e contextos com o intuito de concretizar mais ações e programas que possibilitem atividades de lazer e turismo. O lazer é essencial pois proporciona muitos benefícios como descanso, divertimento, socialização, entre outros que podem melhorar a qualidade de vida (Dumazedier, 1973).

A inserção das PCD nas praias pode trazer benefícios tanto para os usuários quanto para a economia local, constituindo um importante elemento da qualidade de vida do cidadão (Programa praia para todos, 2015). Acredita-se que as PCD necessitam vivenciar momentos prazerosos com igual ou maior intensidade do que os oferecidos pela sociedade (Sassaki, 1999; Sassaki, 2003) a fim de desenvolver as habilidades motoras, a autoestima, a independência, e, principalmente, favorecer a inclusão social (Magnani, 2000; Rocha \& Silva, 2002; Gomes, Rezende, \& Tortorelli, 2010).

No Brasil, existem praias que permitem a possibilidade de um banho de mar a pessoas com deficiência física ou com mobilidade reduzida (Ministério do Turismo, 2006). Algumas prefeituras, em cerca de 10 praias brasileiras, se conscientizaram quanto a problemática da acessibilidade e da inclusão e desenvolveram projetos de facilitação ao banho de mar assistido, apresentando infraestrutura necessária (Governo de Pernambuco, 2015; Projeto Praia sem Barreiras, 2018).

Nesse contexto, o presente estudo prima por estudar um dos projetos destinados a inclusão de PCD e ou com mobilidade reduzida no estado de Pernambuco, é o Projeto Praia sem Barreiras (PSB), que surgiu como uma forma estratégica para implementar mudanças exigidas pela legislação.

O PSB foi criado pela Secretaria de Turismo de Pernambuco (SETUR-PE) e desenvolvido pela Empresa de Turismo de Pernambuco (EMPETUR) em parceria com o Governo do Estado, no ano de 2013. Sua gestão é feita pela Prefeitura da Cidade do Recife, que fornece a infraestrutura e materiais utilizados, com o Centro Universitário Maurício de Nassau (UNINASSAU), que dispõe os acadêmicos dos cursos de Enfermagem, Fisioterapia e 
Educação física, além de um profissional de Fisioterapia que é responsável pela coordenação do projeto (Prefeitura da cidade do Recife, 2013).

O PSB tem como objetivo central permitir o banho de mar assistido e criar uma área de lazer para inclusão e bem-estar por meio de atividades recreativas e esportivas na praia conduzidas por representantes da UNINASSAU: estudantes monitores e professores supervisores.

Para avançar na fronteira do conhecimento sobre o desenvolvimento de competências em Promoção da Saúde nessa ação extensionista interdisciplinar e intersetorial durante a graduação dos cursos de saúde, a presente pesquisa objetivou analisar a compreensão dos usuários do Projeto Praia sem Barreiras sobre as práticas de Promoção da Saúde desenvolvidas pelos estudantes de graduação em saúde.

\section{METODOLOGIA}

Trata-se de um estudo de casos múltiplos incorporados (Yin, 2015), descritivo e exploratório, de natureza qualitativa sobre o aprofundamento teórico e operacional relacionado às vivências dos usuários do Projeto Praia sem Barreiras (PSB) e suas contribuições para a compreensão sobre potenciais competências de Promoção da Saúde baseada nos domínios do Developing Competencies and Profissional Standards for Health Promotion Capacity Building in Europe (CompHP) (Dempsey, Battel-Kirk, \& Barry, 2011) desempenhadas pelos estudantes de graduação em Fisioterapia, Enfermagem e Educação Física de uma Instituição de Ensino Superior (IES) privada.

O grupo de pesquisadores consiste em dois fisioterapeutas, estudantes de Mestrado e docentes da UNINASSAU e um enfermeiro docente de Stricto Sensu, Doutor em Ciências e Mestre em Enfermagem, especialista em Práticas de Promoção da Saúde e Educação e Tecnologias.

A presente pesquisa foi conduzida majoritariamente pela fisioterapeuta, cuja atuação no processo de formação inicial dos docentes envolvidos no PSB para a abordagem com PCD e ou MR foi relevante. À época da coleta de dados, a pesquisadora principal não tinha relacionamento prévio com os participantes.

A pesquisa foi realizada no Projeto Praia Sem Barreiras (PSB), situado na orla da Praia de Boa Viagem, em Recife, PE. O projeto ocupa uma área de $200 \mathrm{~m}^{2}$, cuja estrutura é montada todas as quintas-feiras e desfeita aos domingos, com horário de funcionamento das $8 \mathrm{~h}$ às $12 \mathrm{~h}$ 
(Barbosa, 2014). Para garantir a mobilidade urbana dos participantes do PSB, a Prefeitura criou uma rota acessível desde o bairro de Boa Viagem até a orla de Boa Viagem (Governo do Estado de Pernambuco, 2015).

Realizou-se a coleta do material empírico no período de dezembro/2018 a abril/2019, mediante questionário sociodemográfico de caracterização e entrevista semiestruturada elaborada com perguntas referentes ao tema de estudo, que continha a questão norteadora: Como os usuários do Projeto Praia sem Barreiras compreendem as ações de Promoção da Saúde desenvolvidas pelos estudantes de graduação em saúde que integram o PSB?

No momento em que os usuários chegavam ao PSB, eram abordados pela pesquisadora e se concordassem com a entrevista, esta era realizada em um local reservado, garantindo o sigilo, conforto e segurança.

Foram incluídos no estudo os usuários do PSB da praia de Boa Viagem, de ambos os sexos, maiores de 18 anos, com mobilidade reduzida ou algum tipo de deficiência física, usuários de cadeira de rodas ou não e que frequentam o projeto por pelo menos 3 meses. Foram excluídos aqueles com quaisquer dificuldades de comunicação, alterações cognitivas que dificultasse a compreensão das perguntas realizadas ou que estivessem no projeto apenas de visita.

A entrevista semiestruturada foi guiada por um roteiro com blocos temáticos de questões abertas embasadas pelos domínios de competências em promoção da saúde do CompHP, entendidas como a combinação de conhecimentos, habilidades e valores mínimos e necessários para a prática da promoção da saúde (Pinheiro et al., 2015; Dempsey et al., 2011). As entrevistas foram gravadas em meio digital, transcritas na íntegra com média de 15,8 minutos de duração.

Como referencial teórico foi adotado o Comp HP, instrumento que apresenta 47 competências essenciais para atuação profissional e se divide em nove domínios: (1) possibilidade de mudanças, (2) advocacia em saúde, (3) parceria, (4) comunicação, (5) liderança, (6) diagnóstico, (7) planejamento, (8) implementação e (9) avaliação e pesquisa (Barry et al., 2012; Allegrante et al., 2009), fornecendo um quadro teórico e prático para os profissionais que trabalham com Promoção da Saúde em diferentes contextos e cenários de práticas. 
O processo de análise foi realizado pelos fisioterapeutas de modo integrado e, posteriormente, definidos com apoio do enfermeiro orientador de ambos. Utilizaram-se seis reuniões de supervisão e optou-se por não utilizar softwares para a análise temática do corpus.

Para análise, os dados das entrevistas foram organizados conforme as etapas preconizadas pela técnica de análise de conteúdo de Bardin (2009), com a criação de categorias temáticas de análise de dados por meio da identificação de temas semelhantes. Foram seguidas as etapas: pré-análise, exploração do material (leitura exaustiva do material obtido), tratamento dos resultados (separação dos temas que emergiram em torno de categorias temáticas), inferência/interpretação sobre o fenômeno estudado (Minayo, 2004; Bardin, 2009; Cavalcante, Calixto, \& Bekerr, 2014). Transversalmente, as categorias foram analisadas à luz do referencial teórico, buscando aproximação entre os relatos e as possíveis competências envolvidas

Para registrar observações relativas ao contexto da entrevista, como emoções, expressões verbais, gestuais ou faciais surgidas durante a pesquisa. Adotou-se o Consolidated Criteria for Reporting Qualitative Research (COREQ) para melhorar o rigor, a abrangência e a credibilidade das entrevistas (Tong, Sainsbury, \& Craig, 2007; Equartor Network, 2019).

Os usuários do PSB que participaram da pesquisa assinaram o Termo de Consentimento Livre e Esclarecido (TCLE), destacando-se que o estudo foi realizado considerando-se as observâncias éticas contempladas nas Resoluções 466/12 e 510/16 do Conselho Nacional de Saúde, sendo o projeto de pesquisa aprovado pelo Comitê de Ética em Pesquisa do Centro Universitário Maurício de Nassau - UNINASSAU, conforme parecer consubstanciado $\mathrm{n}^{\circ}$ 2.966.774.

\section{RESULTADOS E DISCUSSÃO}

Participaram do estudo 10 usuários do projeto que foram selecionados por amostra por conveniência, uma vez que a pesquisadora permitiu que todos os usuários interessados participassem das entrevistas até atingir a homogeneidade do grupo baseado no critério de inclusão progressiva (Minayo, 2010). A ausência de temas novos e a repetição de conteúdo nas entrevistas foram indicativos de que as principais ideias já tinham sido levantadas.

Para garantir o anonimato dos participantes, o registro das entrevistas foi feito em um sistema de identificação no qual os nomes verdadeiros foram substituídos pela letra "U" (que 
representa "usuário") seguida de uma numeração cardinal crescente de acordo com a ordem de realização das entrevistas.

Participaram 6 homens (60\%) e 4 mulheres (40\%), metade solteiros, cuja média de idade foi $52,30$ anos (DP $\pm 18,79)$. Quanto à ocupação, sete $(70 \%)$ realizam atividades laborais e estudaram até o ensino médio. Em relação ao tipo de limitação, oito (80\%) eram PCD física e dois $(20 \%)$ com mobilidade reduzida. Metade dos participantes frequenta o Projeto Praia sem Barreiras por mais de 3 anos.

Após transcritas, as falas foram lidas por repetidas vezes com a finalidade de compreender e apreender sentimentos, percepções e emoções do entrevistado. Após a leitura exaustiva, foi realizada a organização dos seus conteúdos de acordo com as categorias que emergiram em cada bloco temático, sendo relacionados dedutivamente ao CompHP (Pinheiro et al., 2015; Dempsey et al., 2011).

Organizaram-se seis categorias temáticas decorrentes das vivências dos usuários do PSB acerca da atuação dos acadêmicos que fazem parte do no projeto. Assim, emergiram 6 categorias temáticas: 1. Dedicação dos acadêmicos atuantes no PSB, 2. Qualificação técnica dos acadêmicos que atuam no PSB, 3. Emoção durante o contato com o mar, 4. PSB promove saúde por melhorar a condição mental, 5. Orientações dadas pelos usuários aos acadêmicos como futuros profissionais, 6. Opiniões acerca do PSB.

\subsection{Dedicação dos acadêmicos atuantes no PSB}

Esta categoria relaciona-se à caracterização dos acadêmicos como atenciosos, educados e dedicados. Os usuários descrevem a maneira como os discentes do PSB recepcionam, interagem com carinho e cuidam de todos.

\footnotetext{
"Estou gostando, são muito comunicativos, atenciosos, nos tratam muito bem, com toda atenção e carinho" (U8).

"O Atendimento dado pelos alunos é de primeira linha, eles nos recebem muito bem para mim é o maior prazer estar aqui no PSB" (U9).

"Eu venho aos sábados. Mas percebo que os alunos são ótimos de tratamento. A dedicação deles eu acho que é o principal, eu só tenho agradecer pelo projeto" (U3).
}

Evidenciam-se os domínios "possibilidade de mudanças" e "comunicação". O domínio "possibilidade de mudanças" manifesta-se pelo fato dos acadêmicos trabalharem em 
colaboração com outros, se unindo para criar ambientes e cenários que promovam a saúde, de modo a facilitar a participação ativa da comunidade (Haeser, Buchele, \& Brozozowski, 2012).

O domínio "comunicação" é exaltado quando a maioria dos usuários do PSB percebe que os acadêmicos possuem habilidades de comunicação que favorecem a participação deles, utilizando de técnicas e meios de comunicação culturalmente adequadas para todos os participantes.

\subsection{Qualificação técnica dos acadêmicos que atuam no PSB}

Os tipos de deficiência e sequelas devem ser apreendidos pelos discentes. Foi percebida nos relatos, certa insatisfação dos usuários quanto à forma de conversarem a respeito da doença e limitações apresentadas e, até mesmo, no momento de ajudá-los e posicioná-los durante as transferências e no banho de mar assistido.

"Eu acho que tem que ter formação para os alunos conhecerem o tipo de deficiência de cada um de nós. Como devem agir para cada tipo, pois cada deficiente tem sua especificidade” (U2).

"Os alunos precisam estar mais atentos porque cada deficiência é diferente. Percebo que nem sempre eles sabem a diferença de um pra outro. Tem gente que não tem equilíbrio de tronco como eu, porém tem pessoas aqui pior que eu. Aí eles botam na cadeira como se a pessoa tivesse equilíbrio, e isso é desconfortável, devem prestar mais atenção nisso" (U5).

"Eu mesmo vou nadando até lá embaixo sozinho, sei nadar muito bem, mas eles não sabiam disso e me tratam como coitadinho. Deveriam perguntar o que a gente precisa, como pode ajudar" (U7).

Acerca deste tema, emergiram características condizentes com a ausência dos domínios "diagnóstico" e "liderança”. O domínio "diagnóstico" torna-se essencial para a compreensão da necessidade dos usuários por meio de métodos quantitativos ou qualitativos com o intuito de analisar o contexto de modo a identificar informações relevantes para que possa ser traçado o plano de ação (Dempsey et al., 2011).

Já o domínio "liderança" é necessário por contribuir para o desenvolvimento de direções estratégicas para a ação em promoção da saúde. Os usuários percebem que os acadêmicos precisam fazer uso de habilidades de liderança para promover o empoderamento e a participação de todos, principalmente do aprendizado da equipe para avançar nas ações de promoção da saúde. 


\subsection{Emoção durante o contato com o mar}

Esta categoria temática se refere à emoção sentida sobremaneira pelos usuários do PSB, uma vez que recordavam de cenas marcantes. A deficiência física e ou mobilidade reduzida implicam em isolamento social, perda de determinados prazeres e restrição ao acesso à praia e outros espaços públicos.

"Agora a cena que me marcou foi a primeira vez que eu vim, aquela ali me marcou, o cuidado que eles tiveram foi imenso, ao me pegar na avenida e me trazer para cá e me levar para a água. Estarem ali conversando comigo, para mim foi sensacional“ (U1).

"Emocionante foi entrar no mar após anos sem conseguir! E através do PSB e da ajuda dos alunos eu ter essa chance de volta [...] é maravilhoso! Sentir a água salgada na boca foi uma sensação indescritível. Tanto emocionou a mim quanto aos estudantes e a todos ao redor, foi muito emocionante“(U4).

"Sentir novamente a água do mar molhando meu corpo, sentir o gosto salgado do mar, essa sensação de leveza e frescor que só o mar proporciona pra gente" (U9).

O domínio "parceria" é percebido nesta categoria temática ao ser identificada a emoção dos usuários por fazerem parte do Projeto Praia sem Barreiras. O PSB se concretizou por existir parcerias entre o Centro Universitário UNINASSAU, Prefeitura da Cidade do Recife e Governo do Estado e dificilmente existiria se não a houvesse.

Tal domínio aborda o trabalho em colaboração com áreas de conhecimento, setores e parceiros para aumentar o impacto e de ações de promoção da saúde (Pinheiro et al., 2015).

\subsection{Projeto Praia sem Barreiras promove saúde por melhorar a condição mental}

A ênfase na sensação de poder (re)criar projetos de vida e formas de cuidar da saúde para ocupar a mente e proteger-se de sentimentos negativos, tais como tristeza maior, preocupações excessivas, ansiedade e sintomas de depressão.

"Eu acho que a saúde começa na mente, quando a gente tem uma mente boa, uma mente que não está pensando besteira, que não vai cair em depressão, a saúde sempre melhora. (...) Eu não digo que aqui é nota dez, é mil porque promove muito a saúde, pois primeiro a saúde começa pela mente e não pelo corpo, a mente é tudo!"(U1).

"A convivência e as histórias que contamos e ouvimos aqui faz com que a gente aprenda e compartilhem com outras pessoas. Isso é bom pra nossa cabeça porque a gente se distrai também, faz a gente se sentir bem" (U3).

"Acho que promovem a minha saúde pelo simples fato de me trazer um motivo para me sentir vivo 
e feliz. Esse projeto fez eu me sentir uma pessoa novamente, me deu vigor, saúde mental. E isso por si só já é contribuir para saúde das pessoas que frequentam esse projeto" (U10).

Saber que o Projeto Praia sem Barreiras promove uma melhora na condição mental dos usuários é um dado extremamente importante para o impacto do projeto a fim de melhorar as ações de promoção da saúde mental e emocional.

\subsection{Orientações dadas pelos usuários aos acadêmicos como futuros profissionais}

Os usuários do PSB mencionaram algumas orientações que desejavam oferecer aos acadêmicos, assim como opiniões para um melhor funcionamento do projeto, como descritos abaixo.

"Através do estudo [...], pesquisar mais e entender a questão da pessoa com deficiência ou com limitações e todos os seus aspectos" (U2).

"Se atualizando, melhorando mais o desempenho, procurar entender cada tipo de deficiência. Quando encontrar o deficiente perguntar o problema dele, se tem alguma limitação. É isso que eu acho que falta neles, não tem esse interesse de perguntar o que aconteceu para eu ter ficado assim. Não vejo esse desempenho" (U5).

"Perguntar a nós, usuários, o que eles podem fazer para facilitar a nossa transferência até o mar porque as pessoas olham pra gente achando que somos coitadinhos, mas pergunte primeiro o que posso fazer? Qual melhor maneira pra você? Posso fazer como? Como você prefere que eu faça?" (U6).

"Eles podem ajudar nos dando orientações quando estão conosco no mar. Conversando sobre a nossa doença, nossa limitação e fornecendo dicas de como ajudar no tratamento e no dia-a-dia" (U10).

Nesta categoria temática identificou-se a ausência do domínio "avaliação e pesquisa". Tal domínio, por meio do uso de métodos de avaliação e pesquisa apropriados, permitiria identificar a insatisfação dos usuários do projeto quando se trata de uma questão técnica que é a forma de posicioná-los e transferi-los para cadeira anfíbia. Entender a doença, suas consequências e saber como lidar com ela, é imprescindível para um promotor de saúde executar suas ações e obter bons resultados.

\subsection{Opiniões acerca do PSB}

A inclusão social, o prazer de voltar a frequentar um local público, a sensação de tomar um 
banho de mar após anos, a melhora da saúde principalmente mental, o divertimento, tudo isso faz com que os usuários do PSB se sintam agradecidos aos acadêmicos de saúde envolvidos.

\footnotetext{
"Não tenho palavras para descrever o que sinto quando estou aqui porque eu tava numa cama achando que não podia fazer muita coisa além de sair do quarto pra sala, mas descobri que posso fazer muito mais, além de tomar um banho de praia tranquilamente“ (U1).

"Que esse projeto veio pra que as PCD e pessoas com dificuldade de locomoção se sintam cidadãos abraçados e amados, como todo pernambucano que mora no litoral“ (U2).

"Só tenho a agradecer a esse projeto que me deu um novo estímulo para vida e me fez acordar. Me fez ver que mesmo com a limitação, posso sim usufruir do lazer e ser frequentador de atividades que nos faz sentir bem" (U8).
}

Ao discorrer sobre as opiniões acerca do PSB, foi percebido que o domínio "parceria" é fundamental para a existência do projeto e responsável por proporcionar sentimentos de gratidão e felicidade. Este domínio é importante por promover benefícios tão importantes na vida das pessoas com deficiência ou com mobilidade reduzida, mediante a um simples banho de mar até a colaboração com diferentes setores e diversos parceiros a fim de promover um impacto positivo na vida dos cidadãos (Pinheiro et al., 2015).

A ausência da triangulação dos dados com os depoimentos de estudantes de graduação (monitores), corpo docente (supervisores) e do currículo da UNINASSAU representa uma limitação do presente estudo. Pretende-se integrar a análise dessas outras fontes de informação em estudos futuros do grupo de pesquisa.

\section{CONSIDERAÇÕES FINAIS}

Os conceitos e os princípios de Promoção da Saúde com foco na criação de oportunidades inclusivas, socializadoras e seguras devem ser trabalhados de modo aprofundado ao longo dos cursos de graduação em saúde, uma vez que os usuários do Projeto Praia sem Barreiras (PSB) enfatizam a positividade da atuação discente nesta ação de extensão universitária interdisciplinar e intersetorial.

Preparar os futuros profissionais de saúde com conhecimentos, habilidades e valores essenciais consiste em uma estratégia potente para promover a acessibilidade de pessoas com deficiência ou mobilidade reduzida a ações de saúde, lazer e cidadania em um ambiente diferencial: as praias, suas orlas e seus arrecifes. 
Portanto, a abordagem qualitativa permitiu explorar o universo de significados dos usuários do PSB em um contexto distinto (exposição solar, segurança das marés, ruído das ondas e do vento ao gravar as entrevistas) e dinâmico (participação nas atividades assistidas por discentes e docentes) a fim de compreender o processo existente e delinear possíveis áreas de fortalecimento das competências de Promoção da Saúde com ênfase na indissociabilidade entre ensino, pesquisa como forma de enfrentamento das iniquidades de pessoas com deficiência ou mobilidade reduzida.

\section{REFERÊNCIAS}

Allegrante, J. P., Barry, M. M., Auld, M. E., Lamarre, M. C., \& Taub, A. (2009). Toward international collaboration on credentialing in health promotion and health education: The Galway Consensus Conference. Heal Educ Behav., 36, 427-38.

Baptista, T. W. F., \& Silva, P. F. A. (2015). A Política Nacional de Promoção da Saúde: texto e contexto de uma política. A National Health Promotion Policy: text and context of a policy. Saúde Debate, 39(esp.), 91-104.

Barbosa, A. F. (2014). Projeto: Praia sem barreiras. Recife: Secretaria de Turismo.

Bardin, L. (2009). Análise de Conteúdo. Lisboa, Portugal, Edições 70.

Barry, M. M., Battel-Kirk, B., Davison, H., Dempsey, C., Parish, R., Schipperen, M. et al. (2012). The CompHP Project Handbooks. Paris (FR): International Union for Health Promotion and Education (IUHPE).

Battel-Kirk, B., \& Barry, M. M. (2013). Developing competency-based accreditation for health promotion in Europe. Rev Med, 92(2), 87-96.

Battel-Kirk, B., Barry, M. M., Zanden, G., Contu, P., Gallardo, C., Martinez, A. et al. (2015). Opertation alising and piloting the IUHPE European accreditation system for health promotion. Glob Health Promot, 22(3), 25-34.

Bezerra, R. M. N. (2014). Acessibilidade. In Dias, Jet al. Novos Comentários à Convenção sobre os Diretos das Pessoas com Deficiência, Brasília, Secretaria de Direitos Humanos da Presidência da República, Secretaria de Promoção dos Direitos da Pessoa com Deficiência.

Brasil. (1988). Constituição da República Federativa do Brasil. Brasília: Senado Federal.

Brasil. (2000). Lei $n^{\circ}$. 10.098, de 19 de Dezembro de 2000. Estabelece normas gerais e critérios básicos para a promoção da acessibilidade das pessoas portadoras de deficiência ou com mobilidade reduzida, e dá outras providências. Diário Oficial [da República Federativa do Brasil], Brasília. 2018. Disponível em: $<$ http://www2.camara.leg.br/legin/fed/lei/2000/lei-10098-19-dezembro-2000-377651-publicacaooriginal-1pl.html>. Acesso em 05 jul 2018.

Brasil. (2004). Decreto № 5.296 de 02 de dezembro de 2004. Regulamenta as Leis nos 10.048 , de 8 de novembro de 2000, que dá prioridade de atendimento às pessoas que especifica, e 10.098, de 19 de dezembro de 2000, que estabelece normas gerais e critérios básicos para a promoção da acessibilidade das pessoas portadoras de deficiência ou com mobilidade reduzida, e dá outras providências. Diário Oficial [da República Federativa do Brasil]. Poder executivo, Brasília-DF. 2004. Disponível em: <http://www.planalto.gov.br/ccivil_03/_Ato2004-2006/2004/Decreto/D5296.htm>. Acesso em:05 jul 2018.

Brasil. (2004). Decreto № 5.296 de 02 de dezembro de 2004. Regulamenta as Leis nos 10.048 , de 8 de novembro de 2000, que dá prioridade de atendimento às pessoas que especifica, e 10.098, de 19 de dezembro de 2000, que estabelece normas gerais e critérios básicos para a promoção da acessibilidade das pessoas portadoras de deficiência ou com mobilidade reduzida, e dá outras providências. Diário Oficial [da República 
Federativa do Brasil], Brasília. Poder executivo, Brasília-DF. 2004 . Disponível em:<http://www.planalto.gov.br/ccivil_03/_Ato2004-2006/2004/Decreto/D5296.htm>. Acesso em: 12 jun 2018.

Brasil. (2014). Ministério da Saúde. Política Nacional de Promoção da Saúde. Brasília, DF: Ministério da Saúde.

Brasil. (2015). Lei Brasileira de Inclusão da Pessoa com Deficiência. Estatuto da Pessoa com Deficiência. № 13.146, 6 de Julho de 2015. Disponível em: <http://www.planalto.gov.br/ccivil_03/_ato20152018/2015/lei/l13146.htm>. Acesso em 21 mai 2018.

Cavalcante, R. B., Calixto, P. P., \& Kerr, M. M. (2014). Análise de Conteúdo: Considerações Gerais, relações com a Pergunta de Pesquisa, Possibilidades e Limitações do Método. Inf \& Soc.:Est., João Pessoa, v.24, n.1, p. 13-18, jan./abr.

Censo Demogrãfico. (2010). Publicado em 20/09/2017. Brasil: IBGE, 2010. Disponível em: <https://censo2010.ibge.gov.br>.Acesso em: 12 jun 2018.

Convenção sobre os direitos das pessoas com deficiência. (2010). Decreto Legislativo $n^{\circ} 186$, de 09 de julho de 2008: Decreto $n^{\circ}$ 6.949, de 25 de agosto de 2009. 4. ed., Rev. e atual. Brasília: Secretaria de Direitos Humanos.

Dempsey, C., Battel-Kirk, B., \& Barry, M. M. (2011). Competências Principais em Promoção da Saúde - CompHP. Versão resumida. Paris: IUHPE.

Dumazedier, J. (1973). Lazer e cultura popular. São Paulo: Perspectiva.

Equator Network. (2017). Diretrizes-chave para relatar os principais tipos de estudos de pesquisa. Oxford. Disponível em: < http://www.equator-network.org/library/resources-in-portuguese-recursos-emportugues/professores/diretrizes-chave-para-relatar-os-principais-tipos-de-estudos-de-pesquisa/>. Acesso em: 08 mai 2019.

Garcia, V. G. (2011). As pessoas com deficiência na história do Brasil. Disponível em: <http://www.bengalalegal.com/pcd-brasil>. Acesso em 02 jun 2018.

Gomes, A. E. G., Rezende, L. K., \& Tortorelli, M. F. P. (2010). Acessibilidade e deficiência: análise de documentos normativos. Cadernos de Pós Graduação em Distúrbios do Desenvolvimento - Universidade Presbiteriana Mackenzie, 10(1), 130-137.

Governo de Pernambuco. (2015). Disponível em: <http://www.pe.gov.br>. Acesso em: 27 mai 2018.

Haeser, L. M., Buchele, F., \& Brozozowski, F. S. (2012). Considerações sobre a autonomia e a promoção da saúde. Physis (Rio J), 22(2), 605-20.

Lanna, J., \& Martins, M. C. (2010). (Comp.). História do Movimento Político das Pessoas com Deficiência no Brasil. - Brasília: Secretaria de Direitos Humanos. Secretaria Nacional de Promoção dos Direitos da Pessoa com Deficiência.

Magnani, J. G. (2000). Lazer, um campo interdisciplinar de pesquisa. In: Bruhns, H. T. Temas sobre o lazer. Campinas: Autores Associados.

Mazzota, M. J. S., \& Dantino, M. E. F. (2011). Inclusão social de Pessoas com Deficiência e Necessidades Especiais: cultura, educação e lazer. Revista Saúde Soc., 20(2), 384.

Minayo, M. C. S. (2010). (org.). Pesquisa social: teoria, método e criatividade. Rio de Janeiro: Vozes, Petrópolis Editora.

Minayo, M. C. S.(2004). O desafio do conhecimento: pesquisa qualitativa em saúde. Rio de Janeiro: Abrasco.

Ministério do Turismo. (2006). Turismo e Acessibilidade: manual de orientações. 2. ed. Brasília: Ministério do Turismo. 
ONU. Convenção Internacional dos Direitos da Pessoa com Deficiência. 2006. Disponível em: <http://www.mp.pe.gov.br>. Acesso em: 13 mai 2018.

Oscip Rodas de Liberdade. Projeto Praia Sem Barreiras. Disponível em: < http://www.turismo.gov.br/\%C3\%BAltimas-not\%C3\%ADcias/7117-praia-sem-barreiras-acessibilidade-atraituristas-com-defici\%C3\%AAncia-para-pernambuco.html>. Acesso em: 06 mai 2018.

Prefeitura da Cidade do Recife. Portal do cidadão. Praia sem Barreiras, 2013 Disponível em: <http://www2.recife.pe.gov.br/servico/praia-sem-barreira?op=NTIzNw>. Acesso em: 02 abr 2018.

Pinheiro, D. G. M., Scabar, T. G., Maeda, S. T., Fracolli, L. A., Peliconi, M. C. F. \& Chiesa, A. M. (2015). Competências em promoção da saúde: desafios da formação. Health promotion competencies: challenges of formation. Saúde Soc., 24(1), 180-188.

Pinheiro, D. G. M., Scabar, T. G., Maeda, S. T., Fracolli, L. A., Peliconi, M. C. F., \& Chiesa, A. M. (2015). Competências em promoção da saúde: desafios da formação. Saúde Soc., 2015, 24(1), 180-8.

Rocha, L. C., \& Silva, W. A. (2002). Tempo e Lazer: Relações com o tempo livre. Rev de Educação Física/UEM, 13(2), 133-139.

Ruschmann, D. S., \& Karina, T. (2006). Planejamento Turístico. Barueri, SP: Manole.

Sassaki, R. K. (1999). A inclusão nos esportes, turismos, lazer e recreação. In: Sassaki, RK. Inclusão: construindo uma sociedade para todos. 3. ed. Rio de Janeiro: WVA, p.91-103.

Sassaki, R. K. (2003). Inclusão: Construindo uma sociedade para todos. 5. ed. Rio de Janeiro: WWA.

Tong, A., Sainsbury, P., \& Craig, J. (2007). Consolidated criteria for reporting qualitative research (COREQ): a 32item checklist for interviews and focus groups. Int J Qual Health Care. 19(6), 349-57.

World Health Organization. (2011). World report on disability. Disponível em:<http://www.unicef.org/protection/World_report_on_disability_eng.pdf>. Acesso em: 11 abr 2018.

Yin, R. K. (2015). Estudo de Caso: Planejamento e Métodos. Bookman Editora. 